\title{
Structural Design and Dynamic Characteristics of Overloaded Horizontal Servo Cylinder for Resisting Dynamic Partial Load
}

\author{
Linan Ma', Qingxue Huang ${ }^{1,2^{*}}$, Lifeng Ma ${ }^{1}$ Qiangjun Ma ${ }^{1}$, Wenze Zhang ${ }^{1}$ and Heyong Han ${ }^{1}$
}

\begin{abstract}
When an output curve force is applied to a horizontal servo cylinder with a heavy load, the piston rod bears a dynamic partial load based on the installation and load characteristics, which significantly affects the frequency response and control accuracy of the servo cylinder. Based on this partial load, increased friction can lead to cylinder bore scuffing, leakage, lack of output power, or even system failure. In this paper, a novel asymmetric static-pressure support structure is proposed based on the principle of hydrostatic support. The radial component force of a dynamic partial load is balanced by cooperation between the support oil cushion of the variable hydraulic pressure support structure, oil cushion of the supportive force, and the damper. Adaptive control of the servo cylinder piston rod, guide sleeve, and piston, as well as the cylinder oil film friction between lubricated surfaces is achieved. In this paper, theoretical design and analysis of the traditional hydrostatic bearing structure and novel structure are presented. A hydraulic dynamic shear scissor is used as a research target to derive a structural dynamic model. Comparative simulations are performed using Matlab Simulink. Additionally, flow field analysis of the novel structure is performed, which verifies the rationality and feasibility of the proposed structure and system.
\end{abstract}

Keywords: Dynamic partial load, Servo cylinder, Asymmetrical static-pressure support structure, Bearing characteristics

\section{Introduction}

The servo cylinder is the executive component of a hydraulic servo system. Its performance directly affects overall system control accuracy under both dynamic and static conditions [1-3]. When an overloaded horizontal servo cylinder articulates an output curve force, a partial load is placed on the piston rod based on the installation and load characteristics $[1,4,5]$, creating friction between the piston rod and guide sleeve, which can lead to leakage and scuffing of the cylinder bore. Additionally, this friction negatively affects the output characteristics and reliability of a servo cylinder, significantly reducing its lifespan [6-8]. Overcoming the influence of dynamic partial loads on a heavy-load horizontal servo cylinder

\footnotetext{
*Correspondence: mqj0220@163.com

${ }^{1}$ Collaboration Innovation Center of Taiyuan Heavy Machinery Equipment, Taiyuan University of Science and Technology,

Taiyuan 030024, China

Full list of author information is available at the end of the article
}

is one of the main goals of servo cylinder structure and servo system design [9-11].

He et al. [12] designed a servo cylinder with an annulargap-throttling hydrostatic bearing to withstand alternating wave loads. Xiao et al. [13] proposed a double-cone hydrostatic bearing for servo-hydraulic cylinders. Zhai [14] designed a novel type of integrated servo-hydraulic cylinder for flight simulators using double-taper hydrostatic bearings. Chen [15] designed a double-stage servohydraulic cylinder using hydrostatic bearings as piston rod seals for a high-speed railway track dynamic testing system. Although most researchers have noted the influence of static pressure on the lubrication and partial load characteristics of servo cylinders, conventional hydrostatic bearing designs for hydraulic cylinder are still widely used [16-18]. However, such designs only solve sealing problems for hydraulic cylinders and cannot solve the partial load problem for servo cylinders. Such cylinders experience heavy loads in horizontal hinged 
installations based on varying swing angles and require more innovative bearing designs. There have been few studies on the dynamic characteristics of hydrostatic servo cylinders. Most of these studies focus on implementing hydrostatic servo cylinder systems as automatic control systems that cannot fully solve the dynamic partial load problem.

Based on this research background, this paper focuses on an overloaded horizontal servo cylinder in a fullhydraulic hobbing shear. The principle of hydrostatic support is used to design a novel type of static asymmetric support for anti-dynamic partial load structures. Dynamic partial load adaptive control is achieved by using a novel control system. A conventional static-pressure structure and the proposed structure are analyzed and a structural dynamic model is derived. The rationality and feasibility of the proposed structure are analyzed by using Matlab Simulink, and the results are verified through experiments.

\section{Dynamic Partial Load Analysis}

The full-hydraulic hobbing shear is a novel steel plate shearing machine that manipulates an upper-shearing edge-rolling steel plate by using a proportional servo valve and displacement sensor to control the position of a double-load servo cylinder $[19,20]$. The structure of this shear is illustrated in Figure 1.

Control of the cutting trajectory and application of sufficient shear force are the key elements for achieving pure-rolling shear of the upper blade on a steel plate. The cutting trajectory and shear force are controlled by the output displacement and output force of two servo cylinders with very high control precision [21-23]. The horizontal hinged heavy-duty servo cylinder output

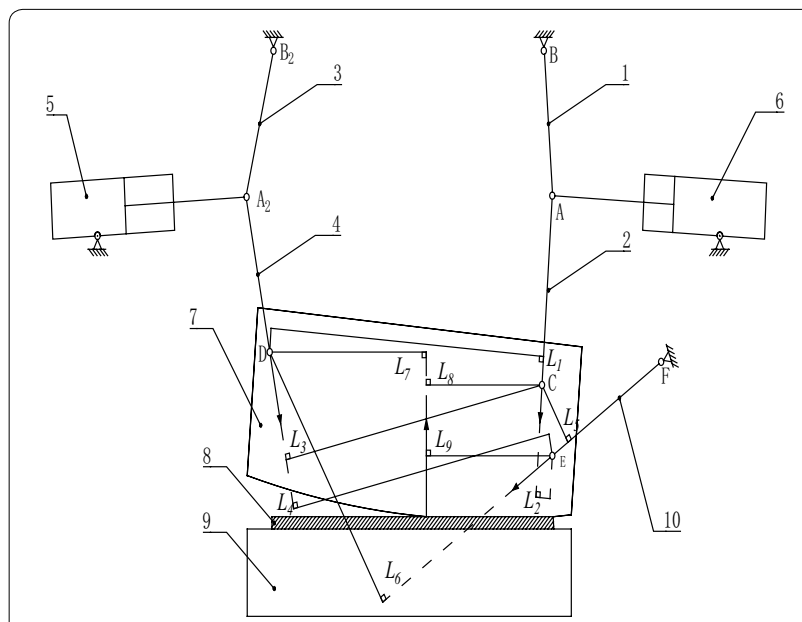

Figure 1 Hydraulic shear mechanism diagram. 1, 3: putter; 2, 4: link; 5, 6: servo cylinder; 7: upper blade holder; 8: steel plate to be cut; 9 : lower blade holder and table; 10: Guide rod force must follow a precise displacement curve and the piston rod must be able to withstand the dynamic partial load. This load produces leakage, cylinder bore scuffing, seal wear, and a lack of output force. These effects lead to piston rod being unable to follow the set trajectory. To satisfy the high-precision control requirements for position, velocity, and force, the ability of the servo cylinders to overcome the influence of partial load on sealing performance and friction loss under heavy-load, high-speed, and high-frequency responses is a key design consideration. Figure 2 presents a photograph of a servo cylinder $[5,24]$.

\section{Design and Principles of an Asymmetrical Support System}

\subsection{Reaction Force Structure and System Design}

A pair of rectangular upper and lower asymmetrical oil pads are installed in the direction of the axis of a servo cylinder. The oil pad on the underside of the piston rod acts as the main support and the oil pad on the upper side of the piston rod acts as a counterforce. Oil is fed under pressure to each oil pad simultaneously. Each oil pad is composed of a central oil inlet, oil chamber, oil seal, and oil return groove. The widths of the support oil pad and counter oil pad are different, as shown in Figure 3.

To be able to adapt to dynamic partial loads caused by radial components and piston self-weight induced by changes in the resultant forces of such radial forces, a damper is installed at the inlet of each oil pad in this design. A separate hydraulic pump supplies pressurized oil to the oil pads and the output pressure Ps of the hydraulic pump is determined by a relief valve and remains unchanged. Figure 4 presents a schematic of this system.

\subsection{Asymmetric Hydrostatic Support Structure Working Principle}

In a servo cylinder, the radial component of a partial load on the piston rod causes the front end of the piston rod to deflect downward relative to the center of mass. This breaks the oil seal between the support oil pad and

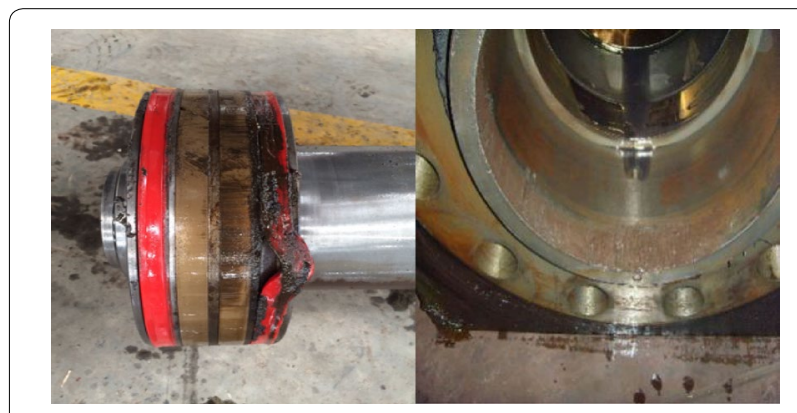

Figure 2 Servo cylinder 


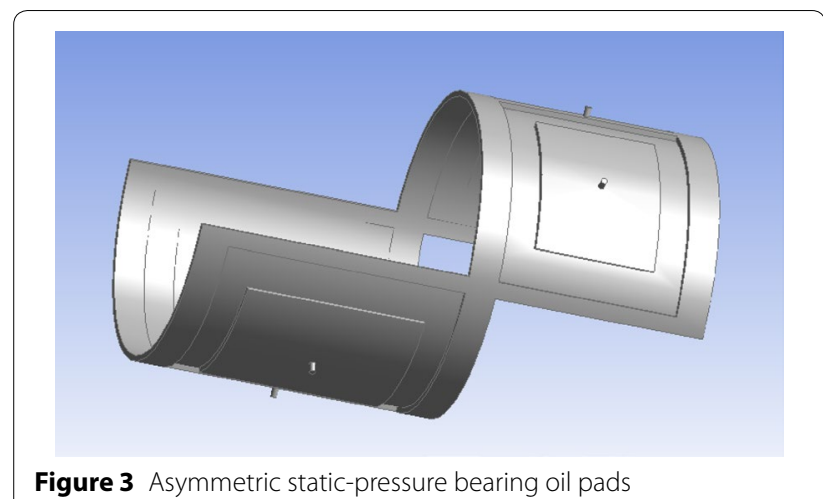

Figure 3 Asymmetric static-pressure bearing oil pads

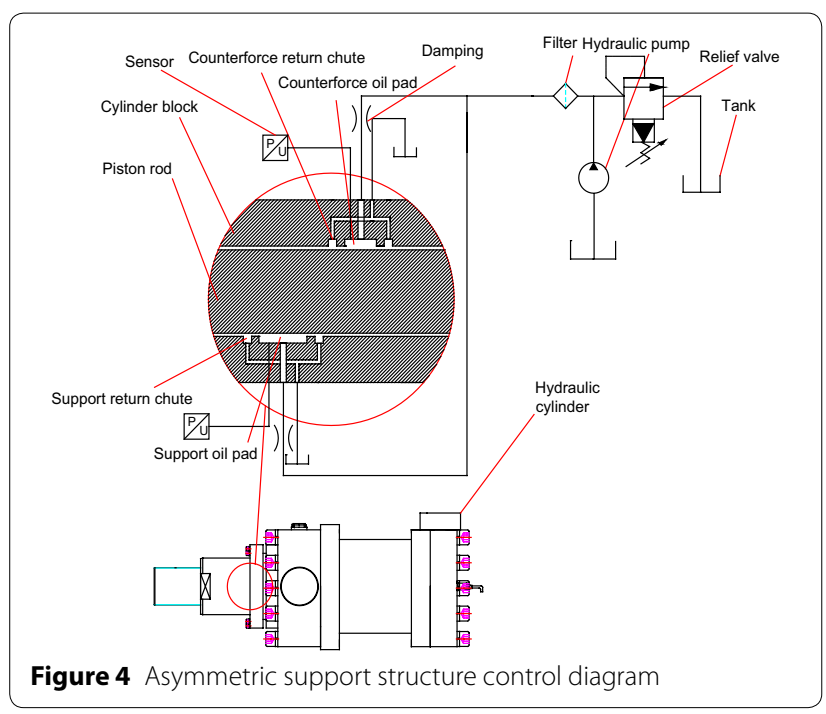

counter oil pad, and changes the piston rod gap, which affects the pressure in the oil chamber of each oil pad. When the radial force increases, based on the centroid location, the gap between the oil seal edges of the piston rod bearing oil cushion and counter oil cushion decreases with increasing deflection of the piston rod. Because the system has a constant-pressure oil supply, under the joint action of hole damping and oil edge sealing, the oil chamber experiences a pressing force. This causes the oil pressure in the upper and lower oil pads of the piston rod to increase, resulting in a larger reaction force. This increased force counteracts the radial force of the eccentric load to balance the partial load. As the movement of the piston rod changes during operation, the radial force generated by the eccentric load also changes dynamically. The gap between the piston rod and oil seal changes correspondingly under the effects of fluid-solid coupling. The pressure in the oil chambers in the upper and lower oil pads then makes the necessary adjustments so the piston rod can be adaptively aligned in the guide sleeve.

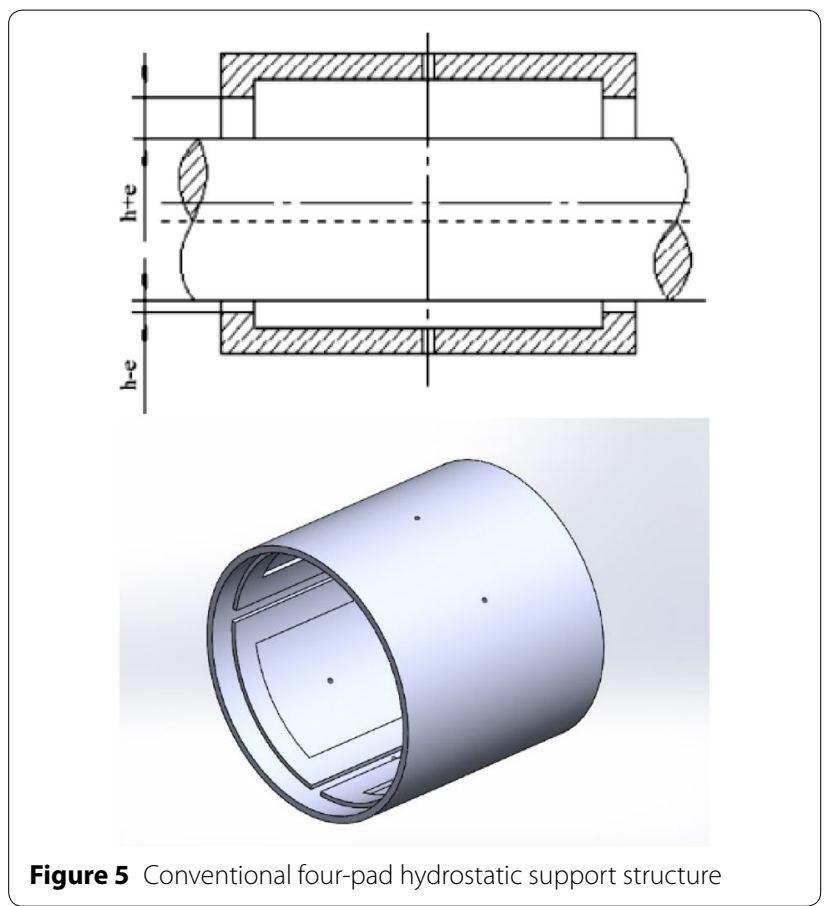

During servo operation, the piston and piston rod are subjected to gravity and partial loading from unbalanced radial components. This force can be adjusted within a certain range using the force generated by pressure changes in the oil chambers of the upper and lower oil pads, which result from changes in the gap between the piston rod and seal oil edge. This ensures that the piston rod, guide sleeve, and cylinder maintain gaps in an appropriate range to facilitate oil film lubrication and reduce friction between the piston rod and guide sleeve, and piston and cylinder. This results in increased system load-bearing capacity and anti-eccentric load capacity, as well as wear-free operation over wide speed and load ranges, which significantly increases the reliability and longevity of horizontal hydraulic cylinders.

\section{Dynamic Model Establishment}

\subsection{Traditional Symmetrical Structure for Static Pressure}

The conventional static-pressure four-pad symmetrical design provides stable bearing pressure to reduce friction between the floating piston rod and guide sleeve during reciprocating motion to improve the overall dynamic performance of a hydraulic cylinder. Based on the pressure difference between the upper and lower symmetry, a radial reaction force is generated on the piston rod to resist displacement in the radial direction [25-27]. Compared to the traditional seal structure, the traditional static-pressure structure (Figure 5) has superior sealing and lubrication characteristics. 


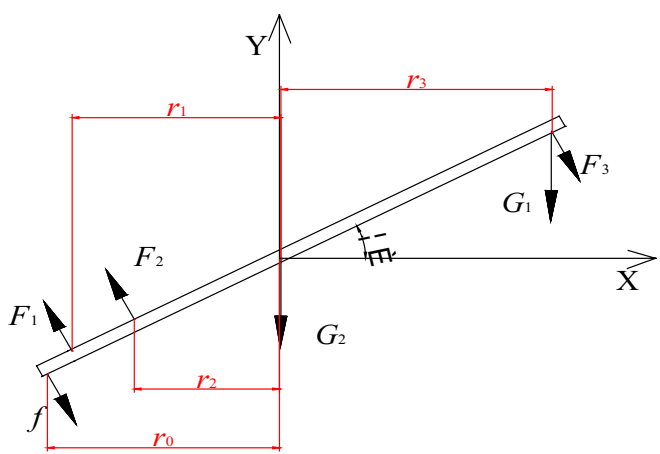

Figure 6 Traditional static-pressure piston rod force analysis

However, this structural design is not suitable for the operation of horizontal articulated heavy-duty servo cylinders with output curve forces. It cannot solve the issues of seal ring wear, cylinder bore scuffing, or a lack of output power. With the goal of applying a traditional staticpressure symmetrical structure to the movement of a horizontal articulated servo cylinder, we performed force analysis on this structure. Figure 6 presents a schematic of the forces acting on a traditional static-pressure piston rod.

In Figure 6, $f$ is the radial component of the partial load, $F_{1}$ is the force between the piston rod and cylinder head guide sleeve, $F_{2}$ is the pressure in the oil chamber of the piston rod hydrostatic support oil pad, $F_{3}$ is the force between the piston and cylinder, $G_{2}$ is the weight of the piston rod, $G_{1}$ is the force of gravity acting on the piston, $\theta$ is the swing angle of the hydraulic cylinder, $r_{0}$ is the distance from the radial force acting point to the piston rod center of mass, $r_{1}$ is the distance from the force $F_{1}$ acting on the piston rod and cylinder head guide sleeve to the piston rod center of mass, $r_{2}$ is the distance from the piston rod bearing oil cushion pressure force $F_{2}$ to the piston rod center of mass, and $r_{3}$ is the distance from the piston center of mass to the piston rod center of mass.

A kinetic model can be obtained through numerical analysis of this system. Eq. (1) represents the out motion of the piston rod and Eq. (2) represents the return motion:

$$
\begin{aligned}
& \left\{\begin{array}{l}
-F_{1} \sin \theta-F_{2} \sin \theta+F_{3} \sin \theta-f \sin \theta=m_{2} \ddot{x}, \\
F_{1} \cos \theta+F_{2} \cos \theta-F_{3} \cos \theta+f \cos \theta-G=m_{2} \ddot{y}, \\
-F_{1} r_{1}-F_{2} r_{2}-F_{3} r_{3}-f r_{0}-G_{2} r_{3} \cos \theta=J \ddot{\theta}, \\
-F_{1}+F_{2}-F_{3}=-f+G_{2} \cos \theta,
\end{array}\right. \\
& \left\{\begin{array}{l}
F_{1} \sin \theta+F_{2} \sin \theta-F_{3} \sin \theta-f \sin \theta=m_{2} \ddot{x}, \\
-F_{1} \cos \theta-F_{2} \cos \theta+F_{3} \cos \theta+f \cos \theta-G=m_{2} \ddot{y}, \\
F_{1} r_{1}+F_{2} r_{2}+F_{3} r_{3}-f r_{0}-G_{2} r_{3} \cos \theta=J \ddot{\theta}, \\
-F_{1}+F_{2}-F_{3}=f-G_{2} \cos \theta,
\end{array}\right.
\end{aligned}
$$

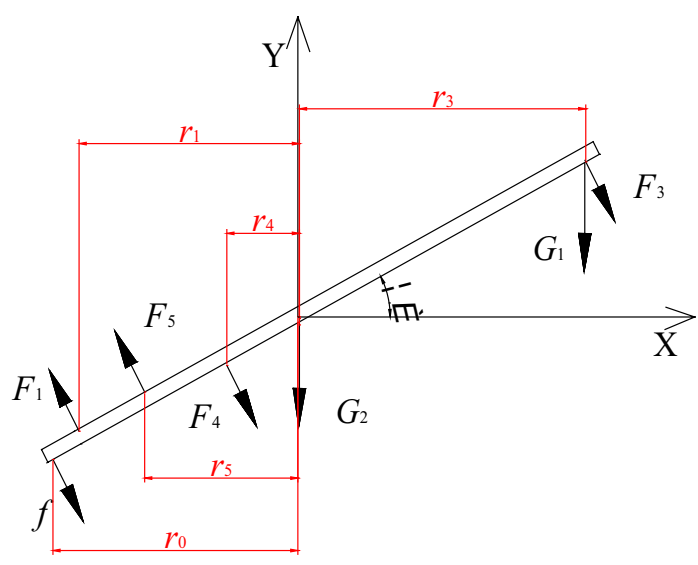

Figure 7 Force analysis of the proposed piston rod with a reactive force structure

where $\ddot{x}$ and $\ddot{y}$ are the acceleration of the cylinder in the horizontal and vertical directions, respectively, $\ddot{\theta}$ is the angular acceleration of the inclusive angle between the cylinder and horizontal axis, $J$ is the moment of inertia of the piston rod, $m_{2}$ represents the quality of the piston rod, and $G$ is the sum of the weight of the piston $\operatorname{rod} G_{2}$ and weight of the cylinder $G_{1}$.

\subsection{Novel Asymmetric Static-Pressure Support Structure}

Figure 3 presented a novel asymmetric support structure for the hydrostatic servo cylinder piston rod shown in Figure 7. In Figure 7, $F_{4}$ is the pressure in the oil chamber of the piston-rod-supporting oil cushion, $F_{5}$ is the oil pressure in the piston-rod-bearing oil pad, $r_{4}$ is distance from the oil pressure force $F_{4}$ of the piston rod to the center of mass of the piston rod, and $r_{5}$ is distance from the oil chamber pressure force $F_{5}$ of the piston rod support oil pad to the center of mass of the piston rod.

According to the stress model of the piston rod, a dynamic mathematical model can be established. The piston particle motion equation is defined as follows:

$$
\left\{\begin{array}{l}
x=r_{3} \cos \theta \\
y=r_{3} \sin \theta
\end{array}\right.
$$

where $x$ and $y$ are the positions of the cylinder particles in horizontal and longitudinal coordinates, respectively.

The acceleration equations for the piston particles in transverse and longitudinal coordinates are defined as follows:

$$
\left\{\begin{array}{l}
\ddot{x}=-r_{3} \ddot{\theta} \sin \theta-r_{3} \dot{\theta}^{2} \cos \theta, \\
\ddot{y}=r_{3} \ddot{\theta} \cos \theta-r_{3} \dot{\theta}^{2} \sin \theta,
\end{array}\right.
$$

where $\dot{\theta}$ is the angular velocity of the inclusive angle between the cylinder and horizontal axis. 
Based on the aforementioned theoretical mechanical equations, we get

$\left\{\begin{array}{l}-F_{1} \sin \theta-F_{5} \sin \theta+F_{4} \sin \theta+F_{3} \sin \theta-f \sin \theta=m_{2} \ddot{x}, \\ F_{1} \cos \theta+F_{5} \cos \theta-F_{4} \cos \theta-F_{3} \cos \theta+f \cos \theta-G=m_{2} \ddot{y}, \\ -F_{1} r_{1}-F_{5} r_{5}+F_{4} r_{4}-F_{3} r_{3}+f r_{0}-G_{2} r_{3} \cos \theta=J \ddot{\theta} .\end{array}\right.$

By simplifying the relationships between the variables, Eq. (5) becomes

$$
\left\{\begin{array}{l}
-F_{1}-F_{5}+F_{4}-F_{3}+f-G_{2} \cos \theta=m_{2} r_{3} \ddot{\theta}, \\
F_{5}=F_{4}-f+G_{2} \cos \theta .
\end{array}\right.
$$

Therefore, a mathematical model for piston rod out motion can be defined as follows:

$$
\left\{\begin{array}{l}
-F_{1} \sin \theta-F_{5} \sin \theta+F_{4} \sin \theta+F_{3} \sin \theta+m_{2} r_{3} \ddot{\theta} \sin \theta \\
\quad=f \sin \theta-m_{2} r_{3} \dot{\theta}^{2} \cos \theta, \\
F_{1} \cos \theta+F_{5} \cos \theta-F_{4} \cos \theta-F_{3} \cos \theta-m_{2} r_{3} \ddot{\theta} \cos \theta \\
\quad=-f \cos \theta-m_{2} r_{3} \dot{\theta}^{2} \sin \theta+G, \\
-F_{1} r_{1}-F_{5} r_{5}+F_{4} r_{4}-F_{3} r_{3}-J \ddot{\theta}=f r_{0}+G_{2} r_{3} \cos \theta, \\
F_{4}-F_{5}=f-G_{2} \cos \theta, \\
-F_{1}-F_{5}+F_{4}-F_{3}-m_{2} r_{3} \ddot{\theta}=f+G_{2} \cos \theta .
\end{array}\right.
$$

Similarly, a mathematical model for piston rod return motion can be defined as follows:

$$
\left\{\begin{array}{l}
F_{1} \sin \theta-F_{5} \sin \theta+F_{4} \sin \theta-F_{3} \sin \theta+m_{2} r_{3} \ddot{\theta} \sin \theta \\
\quad=f \sin \theta-m_{2} r_{3} \dot{\theta}^{2} \cos \theta, \\
-F_{1} \cos \theta+F_{5} \cos \theta-F_{4} \cos \theta+F_{3} \cos \theta-m_{2} r_{3} \ddot{\theta} \cos \theta \\
\quad=-f \cos \theta-m_{2} r_{3} \dot{\theta}^{2} \sin \theta+G, \\
F_{1} r_{1}-F_{5} r_{5}+F_{4} r_{4}+F_{3} r_{3}-J \ddot{\theta}=f r_{0}+G_{2} r_{3} \cos \theta, \\
F_{4}-F_{5}=f-G_{2} \cos \theta, \\
F_{1}-F_{5}+F_{4}+F_{3}-m_{2} r_{3} \ddot{\theta}=f+G_{2} \cos \theta .
\end{array}\right.
$$

By collating (7), a piston rod out motion matrix model can be defined as follows:

$$
\begin{aligned}
& {\left[\begin{array}{ccccc}
-\sin \theta & -\sin \theta & \sin \theta & \sin \theta & m_{2} r_{3} \sin \theta \\
\cos \theta & \cos \theta & -\cos \theta & -\cos \theta & -m_{2} r_{3} \cos \theta \\
-r_{1} & -r_{5} & r_{4} & -r_{3} & -J \\
0 & ; 1 & -1 & 0 & 0 \\
-1 & -1 & 1 & -1 & -m_{2} r_{3}
\end{array}\right]} \\
& \times\left[\begin{array}{c}
F_{1} \\
F_{5} \\
F_{4} \\
F_{3} \\
\ddot{\theta}
\end{array}\right]=\left[\begin{array}{l}
f \sin \theta-m_{2} r_{3} \dot{\theta}^{2} \cos \theta \\
-f \cos \theta-m_{2} r_{3} \dot{\theta}^{2} \sin \theta+G \\
f r_{0}+G_{2} r_{3} \cos \theta \\
-f+G_{2} \cos \theta \\
f+G_{2} \cos \theta
\end{array}\right] .
\end{aligned}
$$

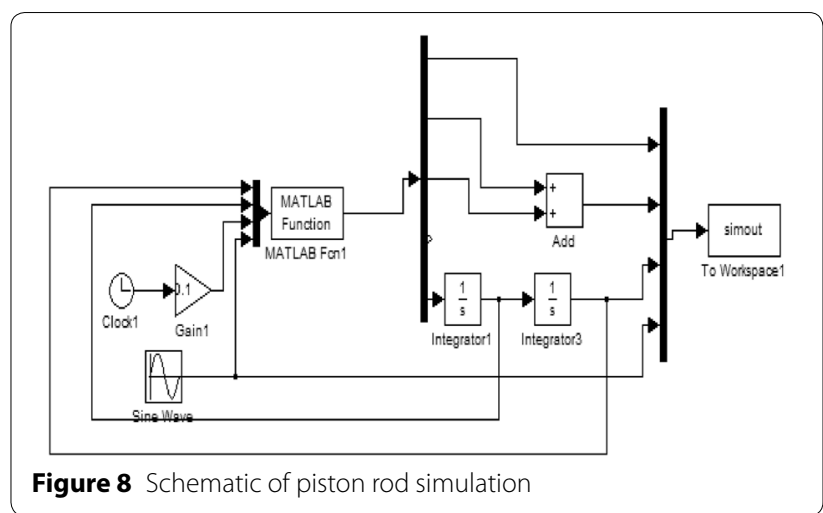

By collating (8), a piston rod return motion matrix model can be defined as follows:

$$
\begin{gathered}
{\left[\begin{array}{ccccc}
\sin \theta & -\sin \theta & \sin \theta & -\sin \theta & m_{2} r_{3} \sin \theta \\
-\cos \theta & \cos \theta & -\cos \theta & \cos \theta & -m_{2} r_{3} \cos \theta \\
r_{1} & -r_{5} & r_{4} & r_{3} & -J \\
0 & -1 & 1 & 0 & 0 \\
1 & -1 & 1 & 1 & -m_{2} r_{3}
\end{array}\right]} \\
\times\left[\begin{array}{r}
F_{1} \\
F_{5} \\
F_{4} \\
F_{3} \\
\ddot{\theta}
\end{array}\right]=\left[\begin{array}{l}
f \sin \theta-m_{2} r_{3} \dot{\theta}^{2} \cos \theta \\
-f \cos \theta-m_{2} r_{3} \dot{\theta}^{2} \sin \theta+G \\
f r_{0}+G_{2} r_{3} \cos \theta \\
f-G_{2} \cos \theta \\
f+G_{2} \cos \theta
\end{array}\right] .
\end{gathered}
$$

\section{Model Simulation}

Hydraulic cylinders with internal diameters of $360 \mathrm{~mm}$, piston rod diameters of $220 \mathrm{~mm}$, and $r_{3}=200 \mathrm{~mm}$ were simulated using the Matlab Simulink commercial software. The piston rod stroke was set to $300 \mathrm{~mm}$. The partial load radial component of the maximum disturbance was set to 2000 N. Figure 8 presents a model simulation chart for the hydraulic cylinder. This model uses a function file to solve equations during simulation.

Figure 9 presents a simulation diagram of the condition where the piston rod is retracted inside the servo cylinder. This model also uses a function file to solve equations.

A sample dynamic model was added to the function file for simulation. Figure 10 presents the changes in hydraulic cylinder swing angle during reciprocation of the piston rod inside the servo cylinder. Figure 11 presents force analysis results for the piston rod during a round-trip motion.

Figure 10 represents the servo cylinder shown in Figure 1 during out motion and return motion. One can see that the servo cylinder swing angle changes over time. 


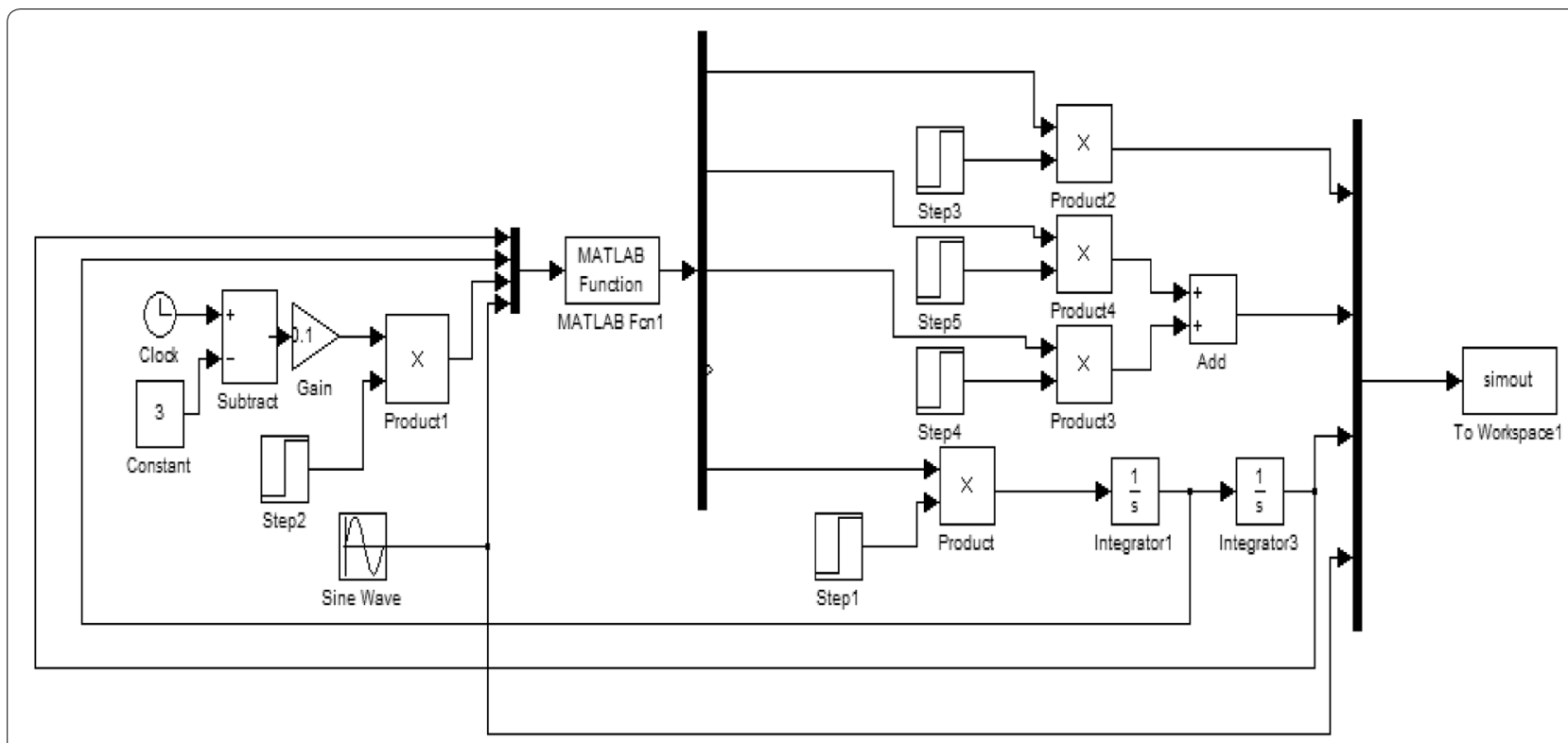

Figure 9 Schematic of piston rod return motion simulation

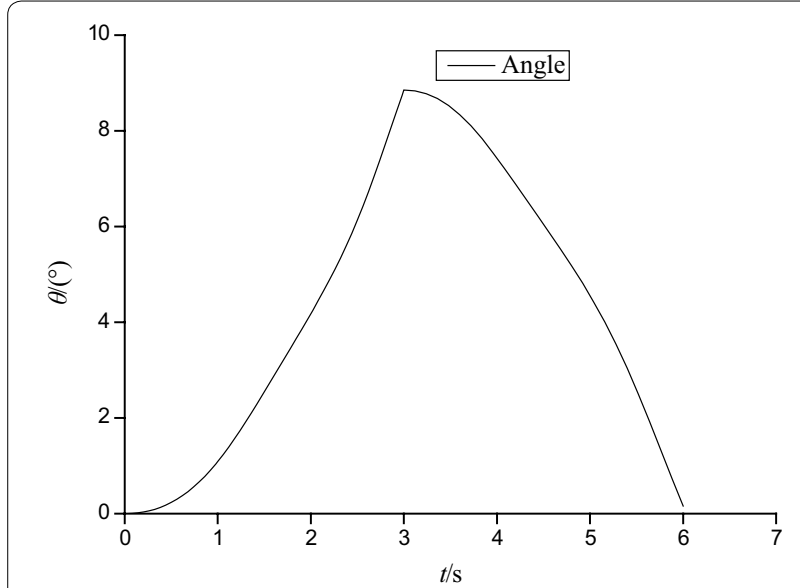

Figure 10 Hydraulic cylinder swing angle

This represents the piston rod output precision displacement curve. In the absence of the proposed asymmetric static-pressure control system, friction occurs between the piston rod and guide sleeve. There is also a lesser degree of friction between the piston and cylinder. Figure 11 presents the piston rod force when the servo cylinder guide sleeve is considered in the asymmetric static-pressure control system. From this figure, one can see that when a dynamic partial load disturbance is added to the front of the piston rod, the resultant force of the asymmetric static-pressure structure is adjusted accordingly such that the force between the piston rod and cylinder head guide sleeve $F_{1}$ approaches zero. Furthermore,

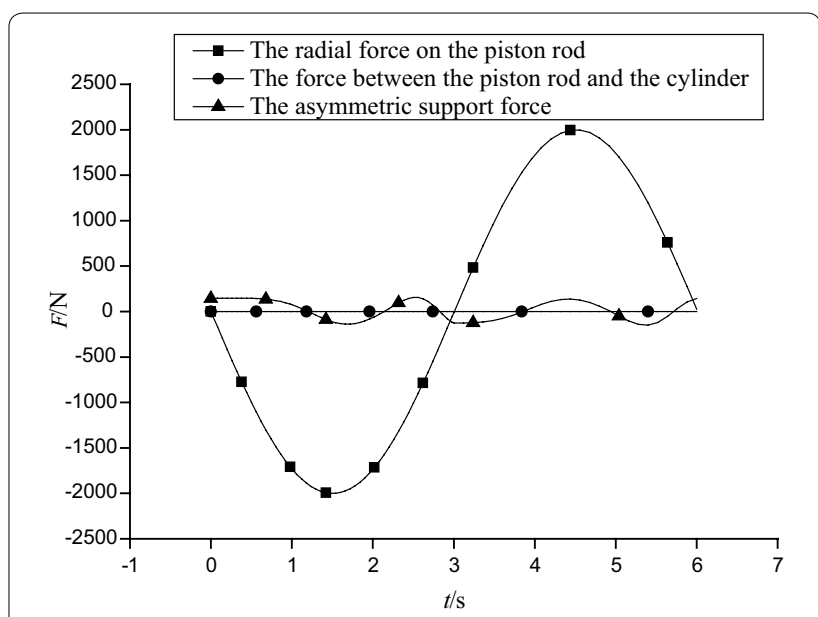

Figure 11 Asymmetric hydrostatic support structure stress simulation

over the entire cycle, the value of $F_{1}$ is very small. During perturbation of the system with a radial component of $2000 \mathrm{~N}$ (see Figure 12), $F_{1}$ reaches a peak value of 50 $\mathrm{pN}$. Because this value is so small, it has no effect on the hydraulic cylinder, meaning the piston rod is completely adjusted during the process of out motion and return motion such that oil film lubrication is achieved without excess friction. This verifies the rationality of the proposed structure and demonstrates that it effectively handles the influence of dynamic partial loads on large servo cylinders. 


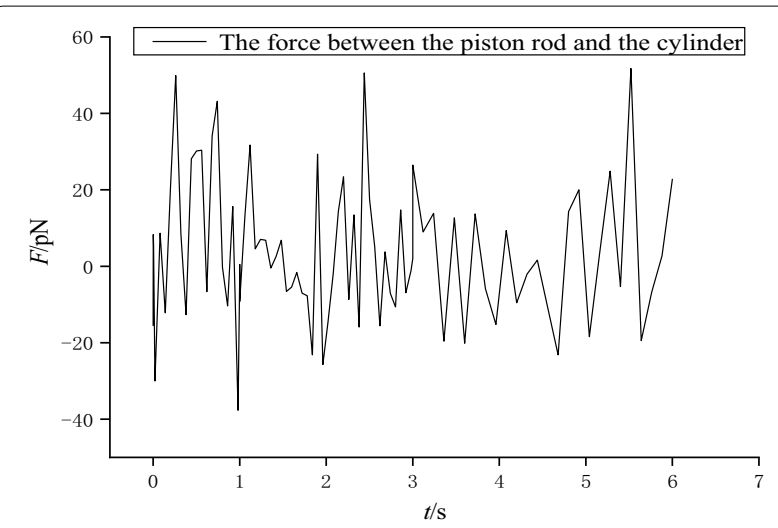

Figure 12 Piston rod and cylinder stress diagram

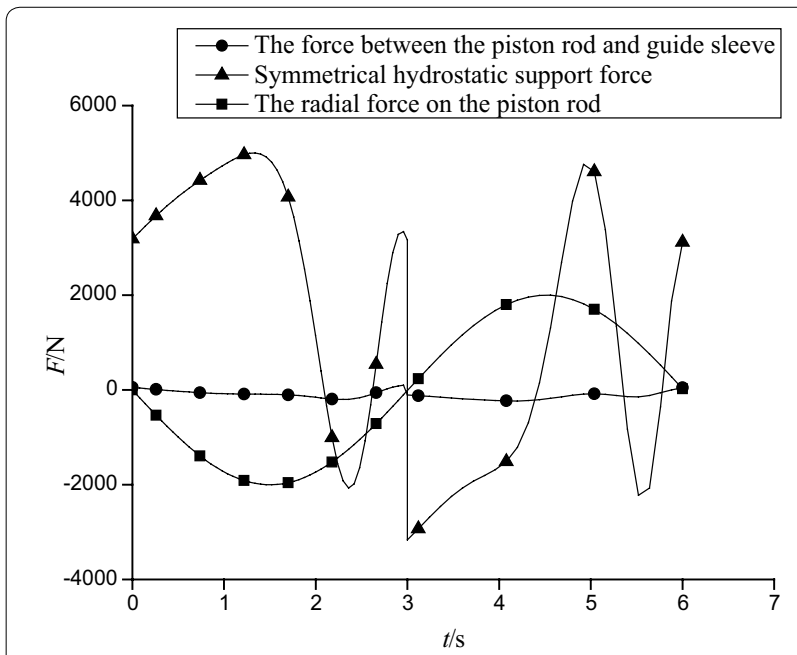

Figure 13 Conventional static-pressure four-pad symmetrical structure force simulation

A dynamic simulation of the traditional static-pressure symmetrical support structure is represented by the force analysis diagram in Figure 13.

From Figure 13, one can see that under the same conditions as those described above, the conventional hydrostatic symmetrical support structure must provide a much greater force when the horizontal articulated servo cylinder is reciprocating. A maximum of $250 \%$ of a given load force is required in response, which is not achievable for this structure. Furthermore, over the entire movement, the direct force fluctuation in the piston rod and guide sleeve is very large, reaching a maximum value of $232 \mathrm{~N}$, which is much higher than the maximum value for the asymmetric hydrostatic support structure design proposed in this paper. From Figure 11, one can see that the response to the maximum eccentric load of $2000 \mathrm{~N}$ for the proposed system is less than $10 \%$ of that value.
The asymmetric static-pressure support structure proposed in this paper can effectively overcome the issues of wear, leakage, and insufficient output force caused by the weight and external load characteristics of a heavyduty servo cylinder with a horizontal hinged installation. From Figure 12, one can see that the direct maximum force between the piston rod and guide sleeve is $50 \mathrm{pN}$ for the proposed structure. Furthermore, by comparing Figures 11 and 13, one can clearly see that the proposed hydrostatic asymmetric support structure is feasible and provides excellent performance.

\section{Flow Field Analysis}

Hydraulic cylinders with full-hydraulic roll shears require output curve forces to perform various tasks. Piston rods are frequently subjected to partial loads, which cause the rods to deflect relative to the center of mass, leading to dry friction with guide sleeves, which reduces the service life of hydraulic cylinders [28-30]. When a piston rod is subjected to a partial load, the two sets of oil pads in the proposed asymmetric hydrostatic support structure generate a corresponding counterforce to balance the partial load on the piston rod and perform automatic centering. The Fluent software package was used to simulate the proposed hydrostatic support structure oil film under partial loading. The simulation results are presented in the following figures. Figure 14 presents a pressure cloud diagram of the oil film of the hydrostatic support structure without a partial load. Figure 15 presents a pressure cloud diagram of the oil film of the hydrostatic support structure under a partial load.

By comparing Figures 14 and 15, one can see that when the piston rod is not subjected to a partial load, the oil is diffused to the periphery of the oil chamber in an elliptical shape. When the piston rod is subjected to a partial load, the oil is diffused into a square shape and the pressure in the oil return groove is low. Additionally, the pressure around the oil chamber gradually decreases and the pressure cloud map changes from orange to yellow, light green, and light blue in sequence. The pressure drop gradient is large and the pressure drop is significant. This is because the gap between the piston rod and sealing oil edge is reduced when the rod is subjected to a partial load. The oil in the oil chamber experiences increased resistance through the return oil gap and the return oil flow rate decreases. Therefore, the pressure in the return oil groove is lower than that without a partial load. The reduced return oil flow causes the pressure in the oil chamber to rise and the oil film pressure drop between the oil chamber and return oil groove increases.

To analyze the influence of partial loading on oil film pressure, the inner-wall surfaces and central surfaces of four oil chambers were considered for pressure 


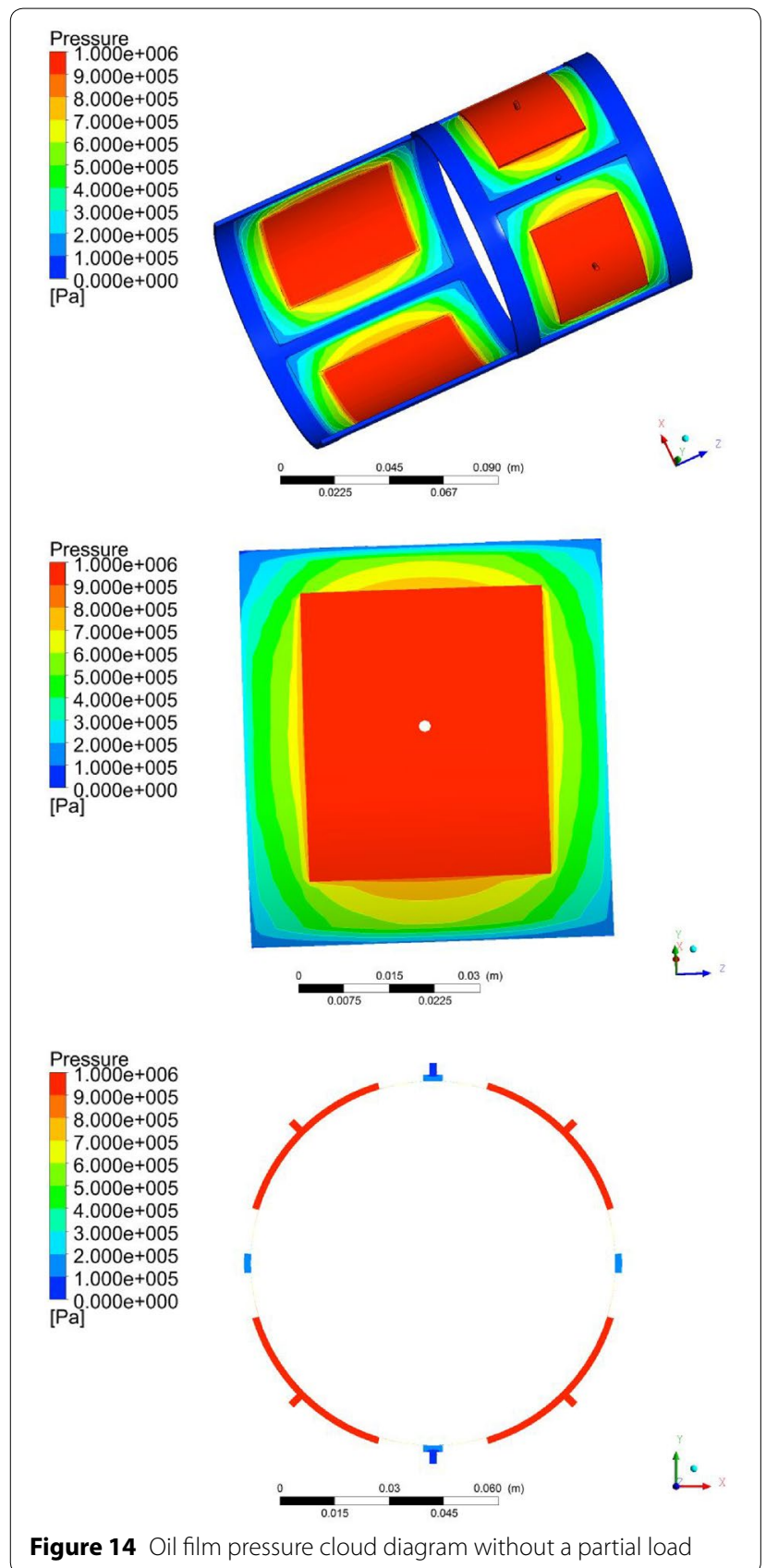

comparison analysis. Figure 16 presents a position-pressure diagram of the inner-wall surfaces of the oil chambers and Figure 17 presents a position-pressure diagram of the central sections of the oil chambers with the $z$-axis as the normal. In Figure 16, the ranges of $30-90 \mathrm{~mm}$ and 120-160 $\mathrm{mm}$ represent the positions of the inner wall surfaces of the oil chambers of the oil pads. The black dots indicate the oil chamber pressure without a partial load with a value of approximately $953275 \mathrm{~Pa}$. The red dots indicate the pressure in the oil chamber with a

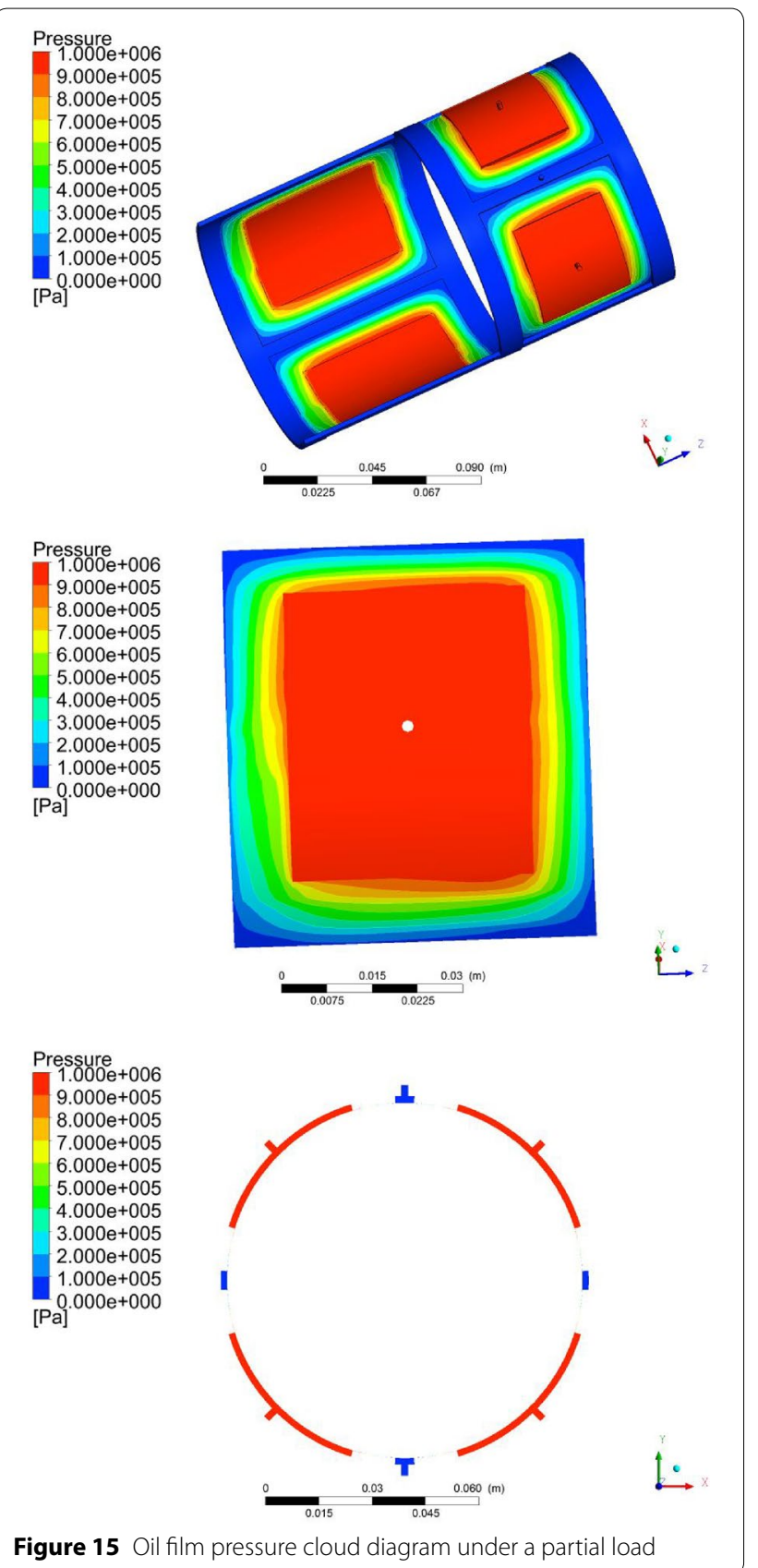

partial load with a value of approximately $998371 \mathrm{~Pa}$. In Figure 17, the black and red dots indicate the pressure at the central sections of the lower oil pads and the upper oil pads, respectively, when the piston rod is subjected to a partial load. The blue and pink dots represent the pressure at the central sections of the lower oil pads and upper oil pads, respectively, when the piston rod is not subjected to a partial load. It is apparent from this figure that when the piston rod is subjected to a partial load, the pressure in the oil chamber is significantly greater than 


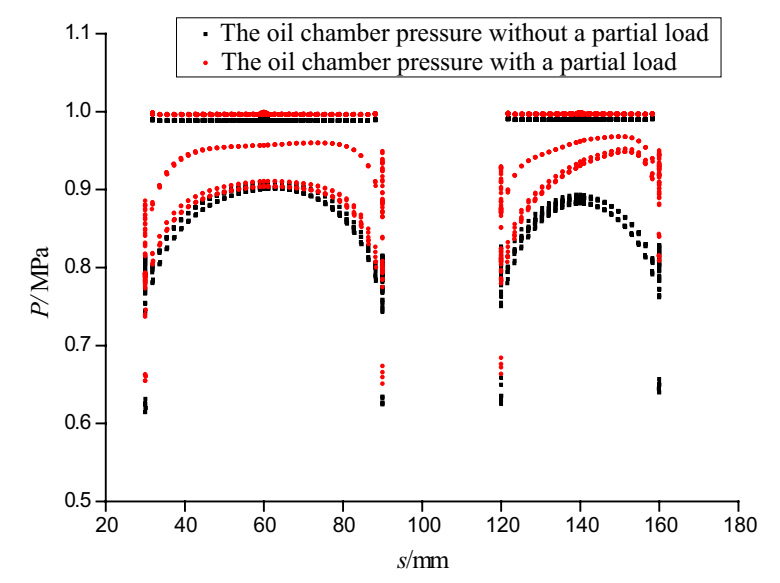

Figure 16 Oil chamber inner-wall position-pressure map

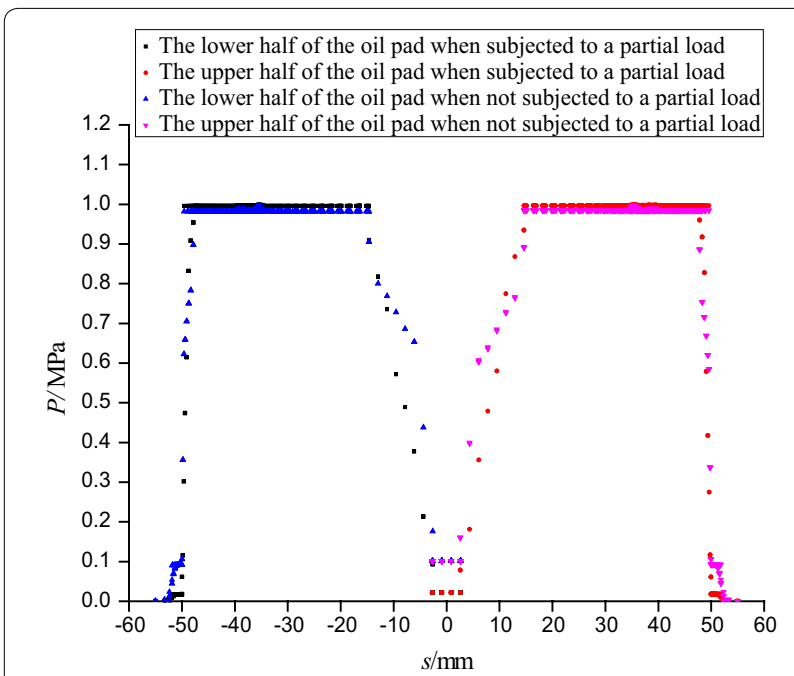

Figure 17 Position-pressure diagram of the central sections of oil pockets with the $z$-axis as the normal

when it is not subjected to a partial load. This is because when the piston rod is subjected to a partial load, the gap between the sealing oil side of the hydrostatic bearing oil pad and piston rod is reduced. Because the system uses a fixed-pressure oil supply, under the joint action of the damper and sealing oil, the oil chamber pressure in the upper and lower oil pads increases to generate a larger reaction force. The increased bearing capacity of the oil chamber is offset by the partial load, thereby achieving the purpose of balancing the partial load. As the movement of the piston rod changes during the working process, the radial force generated by the partial load also changes, which leads to a corresponding change in the gap between the piston rod and sealing oil. The pressure in the oil chambers in the upper and lower oil pads will be adjusted accordingly, meaning the piston rod can achieve self-adaptive balance within the guide sleeve.

\section{Conclusions}

(1) We utilized the static-pressure support principle to design an asymmetric static-pressure structure for heavy-duty horizontal servo cylinder guide sleeves. The proposed structure is equipped with a control system. Based on cooperation between the supporting oil pad, counter oil pad, and damper, the radial component of a dynamic partial load can be balanced to perform real-time centering of the piston rod.

(2) We derived dynamic models for a traditional staticpressure bearing and the proposed counterforce hydrostatic bearing piston rod, and compared simulation results. The simulation results demonstrated that the proposed structure is more suitable for complex working conditions with heavy horizontal partial loads.

(3) Through flow field analysis, we verified that when a servo cylinder is subjected to a partial load, the asymmetric static-pressure supporting force structure can generate a corresponding counterforce to balance the partial load to maintain floating alignment of the piston rod. However, the design and testing of the proposed structure were limited to dynamic load changes in the axial direction of the servo cylinder guide sleeve. Whether or not this structure can adapt to load changes in various directions requires further research to generalize the performance of the structure. In this paper, a structure and servo system design to allow heavy-duty horizontal servo cylinders to overcome dynamic partial loads were presented. This research provides an important basis for theoretical analysis and practical applications in engineering.

\section{Authors' Contributions}

$\mathrm{QH}, \mathrm{LM}$ was in charge of the whole trial; LM wrote the manuscript; $\mathrm{QM}, \mathrm{WZ}$, $\mathrm{HH}$ assisted with sampling and laboratory analyses. All authors read and approved the final manuscript.

\section{Author Details}

${ }^{1}$ Collaboration Innovation Center of Taiyuan Heavy Machinery Equipment, Taiyuan University of Science and Technology, Taiyuan 030024, China. ${ }^{2}$ Taiyuan University of Technology, Taiyuan 030024, China.

\section{Authors' Information}

Linan Ma, born in 1981, is currently a PhD candidate at Collaboration Innovation Center of Heavy Machinery Equipment, Taiyuan University of Science and Technology, China. Her research interests include hydraulic system design and component development. 
Qingxue Huang, born in 1961, is currently an academician of the Chinese Academy of Engineering. His main research interests include Rolling equipment structure optimization design and modern rolling theory and technology research.

Lifeng Ma, born in 1977, is currently a professor and a PhD candidate supervisor at Collaboration Innovation Center of Heavy Machinery Equipment, Taiyuan University of Science and Technology, China. His main research interests include heavy machinery design theory and system complete sets of technology.

Qiangjun Ma, born in 1992, is currently a master candidate at Collaboration Innovation Center of Heavy Machinery Equipment, Taiyuan University of Science and Technology, China. His main research interests include advanced control technology and its application.

Wenze Zhang, born in 1995, is currently a master candidate at Collaboration Innovation Center of Heavy Machinery Equipment, Taiyuan University of Science and Technology, China.

Heyong Han, born in 1982, is currently an associate professor at Collaboration Innovation Center of Heavy Machinery Equipment, Taiyuan University of Science and Technology, China. His main research interests include the electrohydraulic servo system control.

\section{Competing interests}

The authors declare that they have no competing interests.

\section{Funding}

Supported by Nation Youth Science Foundation of China (Grant No. 51505315), Collaboration Innovation Center of Taiyuan Heavy Machinery Equipment and Shanxi Provincial Natural Science Foundation of China (Grant No. 201701D221135), Innovative Project of Graduate Education in Shanxi Province of China (Grant No. 2016BY132).

\section{Publisher's Note}

Springer Nature remains neutral with regard to jurisdictional claims in published maps and institutional affiliations.

Received: 5 June 2018 Accepted: 1 February 2019

Published online: 18 February 2019

\section{References}

[1] B C Liu, L S Yin, H Z Fan, et al. A brief analysis on cause of lateral force in hydraulic cylinder and the counter measures. Construction Machinery and Equipment, 2013(44): 11-14. (in Chinese)

[2] C C Zhan, J H Deng, K S Chen. Research on low-friction and highresponse hydraulic cylinder with variable clearance. Journal of Mechanical Engineering, 2015, 51(24): 161-167. (in Chinese)

[3] H Nabae, M Hemmi, Y Hirota, et al. Super-low friction and lightweight hydraulic cylinder using multi-directional forging magnesium alloy and its application to robotic leg. Advanced Robotics, 2018, 32(9): https:// doi.org/10.1080/01691864.2018.1463868.

[4] Y B Yin, T Tao, KW Zhu, et al. Characteristics of hydrostatic bearing of antiradial load hydraulic cylinder. Fluid Power Transmission \& Control, 2016, 6(79): 12-18. (in Chinese)

[5] Y L Zeng, C J Yao, K S Chen, et al. Simulation and analysis on gap seal of the hydraulic cylinder support guide bush. Wuhan Iron and Steel Corporation Technology, 2017, 55(3): 58-62.

[6] X M Wu, Z L Zhao, SW Zheng, et al. Use of non-contact seal in servo cylinder and its influence on low speed stability of hydraulic system. Machine Tool \& Hydraulics, 2015, 43(13): 72-74. (in Chinese)

[7] F M Jian, ZWWei, Z L Ying. Experimental and theoretical investigation on the sealing performance of the combined seals for reciprocating rod. Journal of Mechanical Science and Technology, 2012, 26(6): 1765-1772.

[8] X Zhao, Z Hu, R Li, et al. Internal leakage fault feature extraction of hydraulic cylinder using wavelet packet energy. Parallel Computational Fluid Dynamics, Springer Berlin Heidelberg, 2014: 363-375.

[9] J M Hale, EY Sim. Self-weight loading of horizontal hydraulic cylinders with axial load. Journal of Physics: Conference Series, 2016, 721(1): https:// doi.org/10.1088/1742-6596/721/1/012006.
[10] L F Lu, C L Chen, L C Zeng, et al. Simulation and characteristics analysis of piston rod hydrostatic support seal for gap seal hydraulic cylinder. Chinese Hydraulics \& Pneumatics, 2012(12): 126-129.

[11] Z B Chu, Y L Yang, Q X Huang, et al. Kinematical analysis of PR-1 1 RIII level composite rodlinkage mechanism. Journal of Sichuan University (Engineering Science Edition), 2015, 47(6): 165-171.

[12] J He, J Zhao, Q X Meng, et al. The design of a new type servo oil cylinder for underwater docking device. Chinese Hydraulics \& Pneumatics, 2006(8): 62-63.

[13] J L Xiao, H Zhou. Application of liquid hydrostatic technology in servo hydraulic cylinder. Machine Tool \& Hydraulics, 1994(1): 43-47.

[14] C R Zhai, R H Zhang. A new type of mechano-electronic integrated servo cylinder used for flight simulator. Machine Tool \& Hydraulics, 1999(1): 13-16.

[15] C J Chen. Simulation of the flow field of hydrostatic support seal for servo hydraulic cylinder based on fluent. Wuhan: Mechanical and Electronic Engineering of Wuhan University of Science and Technology, 2012: 2-2, 8-10, 20-24.

[16] Y Q Wang, X Zhou, Z F Liu, et al. Study on dynamic characteristics of hydrostatic thrust bearing with servo control. Journal of Sichuan University: Engineering Science Edition, 2012, 44(2): 201-205. (in Chinese)

[17] H J Liu, H Guo, S L Zhang. Research on static characteristics of deep/ shallow pockets hybrid, bearing based on FLUENT. Journal of Lubrication Engineering, 2013, 38(10): 35-38. (in Chinese)

[18] YYi, C B Yin, Li Ping, et al. Analysis on characteristics of oil film in hydrostatic support system. Journal of Nanjing Tech University (Natural Science Edition), 2014, 36(2): 35-39. (in Chinese)

[19] HY Han, Q X Huang, L F Ma, et al. Research on the hydraulic system of hydraulic rolling shear. Journal of Sichuan University (Engineering Science Edition), 2011, 43(3): 239-243. (in Chinese)

[20] J B Lei, XY Wang, Y J Pi. Sliding mode control in position control for asymmetrical hydraulic cylinder with chambers connected. Journal of Shanghai Jiaotong University, 2013, 18(4): 454-459.

[21] P Robert, A Harald. Comparison of nonlinear flatness-based control of two coupled hydraulic servo cylinders. IFAC Proceedings Volumes, 2014, 47(3): 10940-10945.

[22] Z L Zhang, S J Wu, JY Zhong, et al. Optimal design of cushion system for high-speed and high-flow valve-controlled hydraulic cylinder. Journal of Central South University (Science and Technology), 2015, 46(10): 3646-3655. (in Chinese)

[23] Z Has, M F Rahmat. Robust tracking control of an electro-hydraulic actuator in the presence of friction and internal leakage. Arabian Journal for Science and Engineering, 2014, 39(4): 2965-2978.

[24] C Cristescu, R Radoi, C Dumitrescu, et al. Experimental research on energy losses through friction in order to increase lifetime of hydraulic cylinders. IOP Conference Series: Materials Science and Engineering, 2017, 174(1): https ://doi.org/10.1088/1757-899x/174/1/012011.

[25] Y Zhou, B Q Sun, S J Wang, et al. Finite element analysis of sealing performance and frictional forces of hydraulic cylinder. Coal Mine Machinery, 2018, 39(03): 50-53. (in Chinese)

[26] Q Li, S Liu, X Pan, et al. A new method for studying the 3D transient flow of misaligned journal bearings in flexible rotor-bearing systems. Journal of Zhejiang University-Science (Applied Physics \& Engineering), 2012, 13(4): 293-310.

[27] Q Li, G Yu, S Liu, et al. Application of computational fluid dynamics and fluid structure interaction techniques for calculating the 3D transient flow of journal bearings coupled with rotor systems. Chinese Journal of Mechanical Engineering, 2012, 25(5): 926-932.

[28] L F Ma, HY Han, Q X Huang, et al. Analysis of commutation impact in hydraulic system of hydraulic rolling shear. Journal of Jilin University (Engineering and Technology Edition), 2012, 42(6): 1396-1401. (in Chinese)

[29] Z B Chu, Q X Huang, L F Ma, et al. Experimental study and simulation of kinetics on linkage structure of rolling-cut bilateral shear. Journal of Sichuan University (Engineering Science Edition), 2011, 43(1): 247-252.

[30] Y Zhou, B Q Sun, S J Wang, et al. Finite element analysis of sealing performance and frictional forces of hydraulic cylinder. Coal Mine Machinery, 2018, 39(3): 50-53. (in Chinese) 